\section{Priapismo secundario a antipsicóticos: a propósito de un caso asociado a risperidona}

\author{
LIZARDO CRUZADO ${ }^{1}$, CÉSAR E. VALLEJOS $^{2}$
}

\section{Priapism associated with risperidone use. Report of one case}

The use of drugs with $\alpha$-adrenergic antagonistic effect is one of the most prominent etiologies of priapism. We report a 32-year-old schizophrenic male in treatment with risperidone who consulted in the emergency room for a painful priapism. A low flow priapism was diagnosed. Medical treatment was unsuccessful and the patient was subjected to a proximal corporo-spongiosal shunt (Quackels technique), with good results. The patient was discharged in good conditions.

(Rev Med Chile 2012; 140: 1445-1448).

Key words: Agents; Antipsychotic; Priapism; Risperidone.

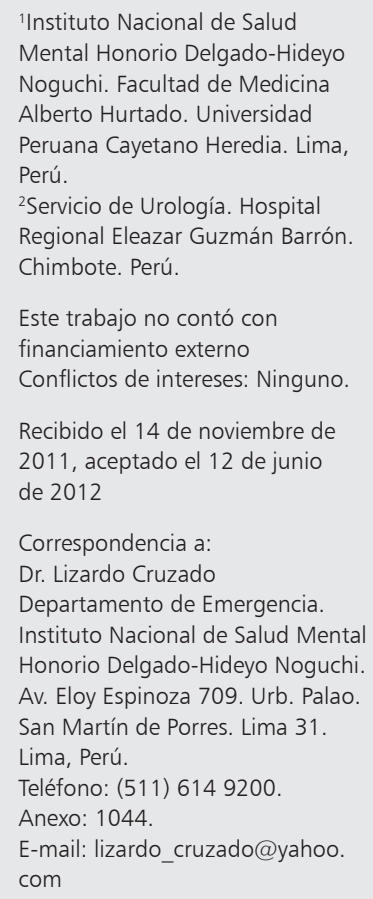

Este trabajo no contó con financiamiento externo Conflictos de intereses: Ninguno.

Recibido el 14 de noviembre de 2011, aceptado el 12 de junio de 2012

Correspondencia a:

Dr. Lizardo Cruzado

Departamento de Emergencia.

Instituto Nacional de Salud Mental Honorio Delgado-Hideyo Noguchi. Av. Eloy Espinoza 709. Urb. Palao. San Martín de Porres. Lima 31. Lima, Perú.

Teléfono: (511) 6149200.

Anexo: 1044

E-mail: lizardo_cruzado@yahoo.

com

$\mathrm{E}$ priapismo es una erección prolongada y usualmente dolorosa que ocurre en ausencia de deseo o estímulo sexual, no se alivia con la masturbación o el coito, y es consecuencia de un desajuste en los mecanismos reguladores que inician la erección peneana y aquellos que permiten su detumescencia ${ }^{1}$. El priapismo se clasifica en dos grupos: el arterial o de alto flujo, menos frecuente, secundario a lesiones que ocasionan entrada de sangre arterial a los cuerpos cavernosos y que no reviste mayor gravedad; y el priapismo de bajo flujo, venooclusivo o isquémico ( $95 \%$ de los casos), que constituye una urgencia urológica por el intenso dolor que ocasiona y porque además puede acarrear consecuencias penosas como la disfunción eréctil irreversible (un importante porcentaje de pacientes la desarrolla, aun con tratamiento) o la gangrena peneana ${ }^{2}$.

Una de las principales causas del priapismo de bajo flujo es el empleo de fármacos con efecto antagonista $\alpha$-adrenérgico, entre los que destacan los medicamentos antipsicóticos, y a los que se atribuyen hasta $50 \%$ de dichos casos ${ }^{3}$. Se asume que el bloqueo adrenérgico desencadena un desequilibrio a favor del tono parasimpático con la consiguiente relajación de las arteriolas cavernosas, disminución del retorno venoso sinusoidal e ingurgitación persistente de los cuerpos cavernosos. Secuencialmente, el incremento de la presión tisular (que en la práctica constituye un síndrome compartimental) llega a superar a la presión arterial con las consiguientes isquemia, hipoxia y acidosis que, al progresar la cascada de eventos, desembocarán en trombosis, muerte celular y fibrosis peneana ${ }^{4}$.

El amplio uso de antipsicóticos en medicina, psiquiatría y otras especialidades obliga a tener en cuenta este infrecuente pero deletéreo evento adverso, que bien puede catalogarse como idiosincrático pues no se relaciona con la dosis del psicofármaco ni con la duración del tratamiento ${ }^{3}$. Con ese fin presentamos y discutimos un caso ilustrativo.

\section{Caso clínico}

Varón de 32 años, soltero, estudiante universitario y deportista profesional, sin otros antecedentes de importancia, con diagnóstico de esquizofrenia paranoide desde hace tres años para lo cual ha recibido tratamiento regular con $3 \mathrm{mg}$ diarios de risperidona. Inició su cuadro de 
priapismo experimentando espontáneamente desde el amanecer -nueve horas antes de ingresar a Emergencia- erección peneana intensa, persistente y dolorosa. Al examen se encontraba quejumbroso, con signos vitales estables y sin otras alteraciones somáticas excepto su notoria erección que respetaba el glande y el cuerpo esponjoso. Entre los exámenes auxiliares cabe anotar hematocrito de $38 \%$ y recuento leucocitario de 10.300 células $/ \mathrm{mm}^{3}$ (sin desviación izquierda), el frotis de sangre periférica fue normal así como las pruebas de coagulación, la bioquímica sanguínea y el urograma. La sangre aspirada de los cuerpos cavernosos mostraba un inequívoco tinte oscuro y su gasimetría arrojó valores de $\mathrm{pH} 6,7 ; \mathrm{pO}_{2}: 15,2$ $\mathrm{mmHg}_{\mathrm{y}} \mathrm{pCO}_{2}: 89 \mathrm{mmHg}$.

Ante los datos referidos y la ausencia de otros factores etiológicos, el caso fue diagnosticado como priapismo de bajo flujo y se proveyó al paciente de medidas de soporte (analgesia, sedación e hidratación), además de efectuarse inyección intracavernosa de fenilefrina diluida en solución salina fisiológica (tres aplicaciones de $0,3 \mathrm{~mL}$ cada una, a concentración de $1 \mathrm{mg} / \mathrm{mL}$ ). Sin embargo, ante el fracaso del tratamiento médico instaurado, a las $22 \mathrm{~h}$ de evolución -el retardo fue debido a no haber disponibilidad de quirófano- se optó por practicar una derivación caverno-esponjoso distal (técnica quirúrgica de Winter). Posteriormente, a las 36 h de observación, y al no verificarse adecuada detumescencia peneana, se tomó la decisión de someter al paciente a una segunda intervención, esta vez con la técnica de Quackels (derivación caverno-esponjosa proximal): en esta ocasión con resultado exitoso pudiéndosele dar de alta.

En el seguimiento ambulatorio se registró presencia espontánea de erecciones nocturnas al mes del alta y a los seis, nuestro paciente lograba la erección de manera parcial y podía efectuar inclusive el coito. Había vuelto además a recibir $2 \mathrm{mg}$ de risperidona y mantenía regular estabilidad en cuanto a su problema psiquiátrico. En la actualidad, cuatro años después del episodio descrito de priapismo, el paciente no presenta disfunción eréctil y su cuadro psicótico es controlado con 450 $\mathrm{mg}$ diarios de quetiapina.

\section{Discusión}

Respecto a la afinidad de los antipsicóticos por el receptor $\alpha 1$-adrenérgico, la lista es encabezada por la clorpromazina, un tradicional neuroléptico fenotiazínico; sin embargo, entre los antipsicóticos atípicos, la risperidona posee una capacidad $\alpha 1$ antagonista muy $\operatorname{similar}^{5}$ (Tabla 1). De hecho, la mayoría de casos de priapismo inducidos por antipsicóticos atípicos son secundarios a risperidona $^{6,7}$-incluyendo recientes casos asociados a su presentación parenteral de depósito Risperdal Consta ${ }^{\oplus}{ }^{8}$. Hasta la fecha, se han reportado casos inducidos por el uso de casi todos los antipsicóticos de segunda generación, incluyendo al aripiprazol ${ }^{9}$. Entre otros fármacos psicotrópicos asociados al desarrollo de priapismo, resalta el antidepresivo e hipnótico trazodona, con una incidencia de hasta un caso de priapismo por cada mil a diez mil usuarios. Inclusive antes de la aparición de los antipsicóticos atípicos, trazodona era responsable de $79 \%$ casos de priapismo inducidos por psicofármacos. Sólo raramente otros antidepresivos se han asociado a priapismo ${ }^{10}$. Cabe hacer notar que, adicionalmente al antagonismo $\alpha 1$-adrenérgico como origen del priapismo, se ha acusado en su desarrollo al disbalance colinérgico/ adrenérgico (incluyendo la neurotrasmisión $\alpha 2$ y $\beta$ adrenérgica), así como la producción aberrante de óxido nítrico y disturbios de la adenosina, algunos factores de crecimiento y ciertas kinasas, entre otros factores complejos ${ }^{2}$.

Recientes revisiones sobre el tópico mencionan un límite de cuatro horas de duración como

Tabla 1. Afinidad de algunos antipsicóticos para el antagonismo de receptores $\alpha 1$-adrenérgicos*

\begin{tabular}{|cc|}
\hline Nombre del antipsicótico & Afinidad** \\
\hline Clorpromazina & 38 \\
\hline Ziprasidona & 38 \\
\hline Risperidona & 37 \\
\hline Tioridazina & 20 \\
\hline Clozapina & 15 \\
\hline Quetiapina & 12 \\
\hline Haloperidol & 6 \\
\hline Aripiprazol & 4 \\
\hline Olanzapina & 2 \\
\hline Pimozida & 1,3 \\
\hline
\end{tabular}

*En base a referencias ${ }^{3,6,7} .{ }^{*}{ }^{*} 10^{-7} \times 1 / K_{d}$, donde $K_{d}$ es la constante molar de disociación en equilibrio. Valores mayores indican más alta afinidad. 
criterio diagnóstico para el priapismo ${ }^{1,2}$. Si bien globalmente el pronóstico depende de la prontitud de la intervención, aun con la instauración del tratamiento específico un alto porcentaje de pacientes -entre 50\% y 90\%- podría desarrollar disfunción eréctil total si el priapismo excede las 24 a 48 h de duración pues, a mayor tiempo transcurrido habrá menor efectividad de cualquier intervención ${ }^{4}$ : esto debido a la abolición de los mecanismos fisiológicos detumescentes y no por falla intrínseca, vale aclarar, de los procedimientos médicos o quirúrgicos instaurados.

Pese a que la incidencia poblacional del priapismo es 1,5 por 100.000 (y se duplica entre los varones mayores de 40 años, que es el grupo etáreo más afectado ${ }^{11}$, la severidad de las posibles secuelas impone reforzar la actitud preventiva ante la posibilidad de esta complicación: en el caso del priapismo inducido por antipsicóticos, medicamentos comúnmente de uso crónico, una medida preventiva elemental es detectar la presencia de factores de riesgo adicionales como son, entre otros: discrasias sanguíneas (policitemia, talasemias, drepanocitosis), consumo de sustancias psicoactivas (alcohol, cocaína, anfetaminas), empleo de otros fármacos $\alpha$-bloqueadores (terazosina, tamsulosina, guanetidina, etc. $)^{1,2}$, que podrían desaconsejar el uso de antipsicóticos con alta afinidad $\alpha$-antagonista como la risperidona ${ }^{6} \mathrm{o}$, en caso de su prescripción, imponer obligadamente una monitorización cercana. También se deben considerar posibles interacciones farmacocinéticas que eleven los niveles plasmáticos de los fármacos asociados con priapismo ${ }^{13}$. Adicionalmente se ha descrito que numerosos pacientes desarrollan erecciones prolongadas y no dolorosas antes del episodio pleno de priapismo: sin embargo, los pacientes no mencionan espontáneamente este problema (y tampoco lo abordan usualmente los facultativos $)^{11}$. En ese sentido, se debiera interrogar sistemáticamente a los usuarios por la presencia de estos fenómenos durante el tiempo de empleo del fármaco y, de haber respuesta positiva, considerar oportunamente su posible suspensión.

El abordaje terapéutico del priapismo depende fundamentalmente del tipo diagnosticado: en el caso del priapismo isquémico, el manejo inicial implica aspiración e irrigación directa de los cuerpos cavernosos con agentes simpaticomiméticos diluídos - por ejemplo, fenilefrina o etilefrina- $y$, en caso de fracasar estos procedimientos, se deberá efectuar la anastomosis quirúrgica de los cuerpos cavernosos hacia el cuerpo esponjoso o afluentes venosos -como la vena safena mayor- a fin de lograr la flacidez peneana: ya sea la anastomosis efectuada a nivel del glande o, en casos más severos, incluso en la base del pene. La eficacia de estos procedimientos para conservar la función eréctil no es absoluta, como se ha mencionado ${ }^{1,2,4}$.

En el presente caso verificamos la imprevisibilidad del priapismo -que no dependió de la dosis prescrita de risperidona, más bien baja, ni del tiempo de administración- ${ }^{9}$. Probablemente la edad juvenil y el buen estado físico de nuestro paciente haya influido en la retención de su capacidad coital; empero, es imprescindible considerar la posibilidad de recurrencia del priapismo y prevenirlo de acuerdo a los factores de riesgo que involucran, en primer término, la afinidad del antipsicótico escogido hacia el bloqueo $\alpha 1^{5,7}$. Se ha reportado recurrencia del priapismo en casos de reexposición al antipsicótico previamente prescrito?.

La pérdida o menoscabo de la virilidad usualmente acarrea sentimientos de culpa, ansiedad, vergüenza y depresión, casos en los que suele requerirse intervención psiquiátrica adicional ${ }^{3}$. En función de lo reseñado, hacemos hincapié en la necesidad de prevenir el priapismo asociado a antipsicóticos, dentro de lo posible, y la perentoriedad de la intervención urológica temprana para evitar indeseables desenlaces sobre la capacidad eréctil de nuestros pacientes.

\section{Referencias}

1. Broderick GA, Kadioglu A, Bivalacqua TJ, Ghanem H, Nehra A, Shamioul R. Priapism: pathogenesis, epidemiology, and management. J Sex Med 2010; 7: 476-500.

2. Burnett AL, Bivalacqua TJ. Priapism: New concepts in medical and surgical management. Urol Clin N Am 2011; 38: 185-94.

3. Compton MT, Miller AH. Priapism associated with conventional and atypical antipsychotic medications: a review. J Clin Psychiatry. 2001; 62: 362-6.

4. Kadioglu A, Sanli O, Celtik M, Cakan M, Taskapu H, Akman T. Practical management of patients with priapism. EAU-EBU update series 2006; 4: 150-60.

5. Sood S, James W, Bailon MJ. Priapism associated with atypical antipsychotic medications: a review. Int Clin Psychopharmacol 2008; 23: 9-17.

6. Makesar D, Thome J. Risperidone-induced priapism. World J Biol Psychiatry 2007; 8: 45-7. 
Priapismo secundario a antipsicóticos - L. Cruzado et al

7. Andersohn F, Schmedt N, Weinmann S, Willich SN, Garbe E. Priapism associated with antipsychotics. Role of $\alpha 1$ adrenoceptor affinity. J Clin Psychopharmacol 2010; 30: 68-71.

8. Kirshner A, Davis RR. Priapism associated with the switch from oral to injectable risperidone. J Clin Psychopharmacol 2006; 26: 626-8.

9. Wang CS, Kao WT, Chen CD, Tung YP, Lung FW. Priapism associated with typical and atypical antipsychotic medications. Int Clin Psychopharmacol 2006; 21: 245-8.

10. Weiner DM, Lowe FC. Psychotropic drug-induced priapism. Incidence, mechanism and management. CNS Drugs 1998; 9: 371-9.

11. Eland IA, Lei J, Stricker BH, Sturkenboom MJ. Incidence of priapism in the general population. Urology 2001; 57: 970-2.

12. Freed M, Muskin PR. Getting patients to talk about priapism. Curr Psychiatr 2003; 2: 61-2, 67-8, 70.

13. Geraci MJ, McCoy SL, Crum PM, Patel RA. Antipsychotic-induced priapism in an HIV patient: a cytochrome P450-mediated drug interaction. Int J Emerg Med 2010; 3: 81-4. 